539. 22 : 539. 389.2

\title{
Deformation of Polycrystalline Metal Composed of Anisotropic Crystals Having Various Stress-Strain Curves*
}

\author{
By Takeji ABE**
}

The effect of stress-strain curve on the deformation behavior of polycrystalline metal composed of anisotropic crystals is investigated from energy aspect. The constraint between grains is discussed in the cases that the stress-strain relations are represented by (1) combination of straight lines, and (2) exponential curve.

It is pointed out that the mutual constraint between grains is affected by the shapes of stress-strain curves, as well as the anisotropy or the distribution of orientations of grains. It is also concluded that the deformation of polycrystals changes its mode from constant stress to constant strain as the total strain becomes greater and as the grain size becomes smaller.

\section{Introduction}

In the previous paper(1), the deformation behavior of a polycrystalline metal composed of anisotropic crystals having a linear stressstrain relation was analysed from energy aspect. The linear stress-strain relation considered in the previous paper corresponds to the elastic deformation of metals. The stress-strain relation in plastic range, however, is more complicated in general. For instance, it is known experimentally that the plastic stress-strain relation is approximately represented by an exponential function for most metals.

In order to apply the energetic consideration to the plastic deformation of metals, the effect of stress-strain function on the deformation behavior of a polycrystalline metal is investigated in the present paper. Two cases where the stress-strain relations are represented by (1) combination of straight lines, and (2) exponential curve are studied. Particularly, the restriction between grains is discussed.

These considerations will be of great use in discussing (a) the correlation between the deformation behaviors of a single crystal and polycrystals, (b) the meaning of residual stress

* Received 15th October, 1969.

** Assistant Professor, Faculty of Engineering, Kyoto University, Sakyo-ku, Kyoto. measured by $\mathrm{X}$-ray, and (c) the condition of microscopic crack initiation in the heavy strained region. In the following analysis, a polycrystalline metal is expressed by a model composed of a pile of cubics as shown in Fig. 1. We consider representatively the grains lying perpendicular to the stress axis shown with a thick line in Fig. 1.

\section{Basic relations}

In the previous paper ${ }^{(1)}$, the basic relations for the polycrystals having a stress-strain relation expressed by straight lines starting from the origin were derived (Fig. 2).

Namely,

$$
\sigma=K \varepsilon
$$

Some of the equations obtained are reviewed below, for they are necessary in the analysis
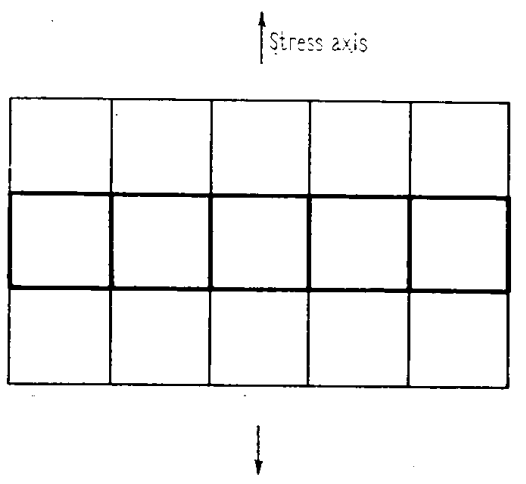

Fig. 1 Model of polycrystals 
afterwards.

\section{$2 \cdot 1$ Stress and strain}

Stress $\sigma$ and strain $\varepsilon$ in an arbitrary grain are expressed respectively with

$\sigma=\tilde{\sigma}+\Delta \sigma, \quad \varepsilon=\tilde{\varepsilon}+\Delta \varepsilon$

where the top wavy line $\sim$ expresses the averaged value for all grain orientations.

$$
\tilde{\sigma}=\int \sigma d \Omega, \quad \tilde{\varepsilon}=\int \varepsilon d \Omega
$$

$\int d \Omega$ expresses the normalized angular integra$\operatorname{tion}\left(\int d \Omega=1\right)$.

\section{$2 \cdot 2$ Constraint ratio $\boldsymbol{\eta}$ between grains}

The constraint ratio $\eta$ between grains is defined by

$$
\eta=\Delta \varepsilon / \Delta \varepsilon_{\max }
$$

as shown in Fig. 2. Considering from the physical meaning of $\eta$, it is necessary that the range of $\eta$ be taken as $0 \leqq \eta \leqq 1$. It is obvious from Fig. 2 that,

$$
\left.\begin{array}{l}
\eta=0: \text { constant strain } \\
\eta=1: \text { constant stress }
\end{array}\right\}
$$

In the following analysis, it is assumed for the first approximation that the value of $\eta$ is independent of the grain orientations, i.e. $\eta=$ constant. Then, the trajectory of the position of each grain on the stress-strain diagram after deformation becomes a curve in general, as shown in Fig. 2. Let us define the inclination of the curve at the intersecting point with the line of $\widetilde{K}$ by $K^{\prime}$.

$$
K^{\prime}=-\frac{(1-\eta)}{\eta} \widetilde{K}
$$

where $\widetilde{K}$ is the mean value of $K$ for all grains and given by ${ }^{(1)}$

$$
\widetilde{K} \cong \tilde{K}_{\sigma}+(1-\eta) \frac{\widetilde{\left(\Delta K_{\varepsilon}\right)^{2}}}{\widetilde{K}_{\varepsilon}}
$$

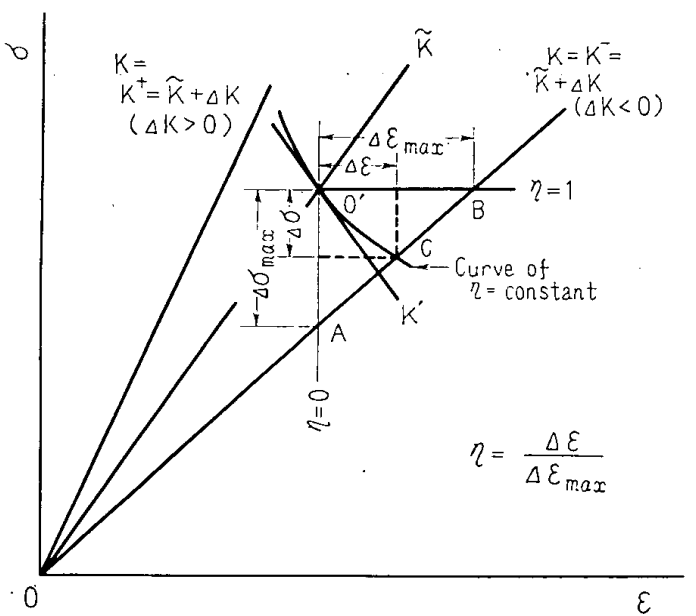

Fig. 2 Relation between $K, \eta, \Delta \sigma, \Delta \varepsilon$ and $K^{\prime}$ on stress-strain diagram
The subscripts $\sigma$ and $\varepsilon$ express the values under the conditions of constant stress and constant strain, respectively. $\left(\widetilde{\left.\Delta K_{\varepsilon}\right)^{2}}\right.$ in Eq. $(7)$ is given by

$$
\widetilde{\left(\Delta K_{\varepsilon}\right)^{2}}=\int\left(\Delta K_{\varepsilon}\right)^{2} d \Omega
$$

\subsection{Deformation energy $W$}

In order to obtain the deformation energy of polycrystals, let us assume two grains having inclinations $K^{+}$and $K^{-}$on the stress-strain diagram and define their deformation energies by $W^{+}$and $W^{-}$, respectively. At first, a case of no constraint between the grains is considered. The deformation energy $W^{\prime}$ of a system composed of two grains is given by

$$
W^{\prime}=\frac{1}{2}\left(W^{+}+W^{-}\right)
$$

(c.f. Fig. 7). The energies treated in the present paper are all for unit volume. The deformation energy $W$ for polycrystals is given by

$$
W=\int W^{\prime} d \Omega
$$

Next, the increase in energy owing to the mutual restriction between the grains is taken into account. (For the first approximation, only

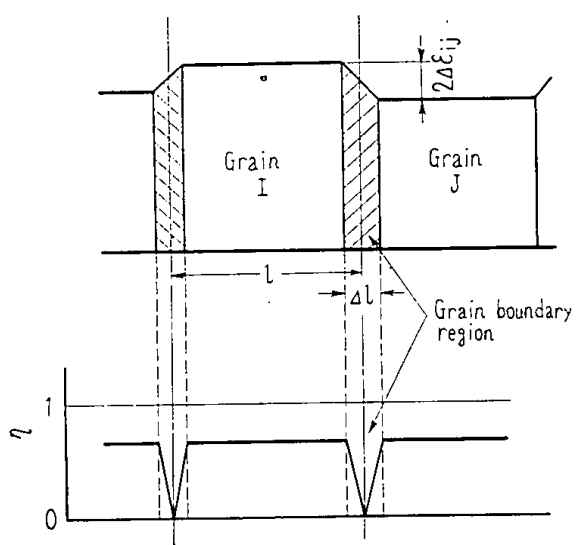

Fig. 3 Distribution of strain and constraint ratio. in grain boundary region

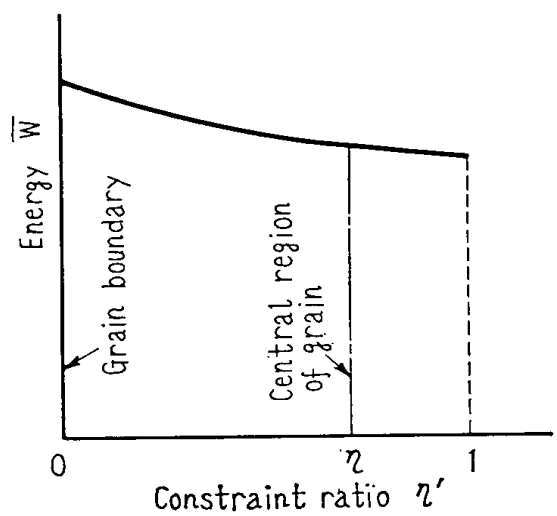

Fig. 4 Increase of energy at grain boundary region (Schematic) 
the restriction by the adjacent grains is analysed below, for it is considered to be most effective constraint during the deformation of polycrystals.) Let us suppose two neighboring grains, I and $\mathrm{J}$, having the inclinations $K_{i}$ and $K_{j}$ on the stress-strain diagram, respectively. The difference $\Delta K_{i j}$ of the inclinations is defined by

$$
2 \Delta K_{i j}=K_{i}-K_{j}
$$

The strain is continuous across the grain boundary, that is, the strains in the neighboring grains are equal at the boundary between them. Therefore, $\eta=0$ at the boundary (Fig. 3 ). The relation between the energy $\bar{W}$ and the constraint ratio $\eta$ of a system composed of two grains is schematically shown in Fig. 4. The head straight lin - eexpresses the mean value of each symbol for the system of two grains. The energy increase $\Delta W$ in the grain boundary region corresponding to the decrease of the constraint ratio from $\eta$ to $\eta^{\prime}$ is given by

$$
\Delta W=\bar{W}\left(\eta^{\prime}\right)-\bar{W}(\eta)
$$

If we define the width of the grain boundary region by $A l$ (Fig. 3 ), the ratio $P$ of the grain boundary region is expressed by

$$
P=\Delta l / l
$$

where $l$ is the width of grain. The value of $P$ is considered to be dependent on $\eta$ in general. So, let us assume that

$$
P=\eta P_{1}
$$

where $P_{1}$ is the value of $P$ under the constant stress condition $(\eta=1)$. It is also Supposed that the distribution of the strain in the grain boundary region is linear as schematically shown in Fig. 3, and that the value of $\eta$ changes linearly in that region as is evident from Eqs. (2) and (4). The deformation energy $W b^{\prime}$ in the grain boundary region per unit volume is given from Eqs. (12) and (14), as follows

$$
W_{b}^{\prime}=2 P_{1} \int_{0}^{\eta} \Delta W d \eta^{\prime}
$$

The energy $W_{b}$ in the grain boundary region for all the polycrystals is given by

$$
W_{b}=\int W_{b^{\prime}} d \Omega
$$

The total deformation energy $W_{t}$ of polycrystals including the restriction at the boundary is given from Eqs. (10) and (16) by

$$
W_{t}=W+W_{b}
$$

The value $\eta$ which minimizes $W_{t}$ is obtained from

$$
\partial W_{t} / \partial \eta=0
$$

In the following chapters, discussions are made on the influence of the shape of stress-strain curve on the deformation mode of polycrystals, based on the equations mentioned above.

\section{Deformation of polycrystals having stress-strain relation expressed with combination of straight lines}

3.1 The case that the stress-strain relation is expressed by

$$
\sigma=\sigma_{0}+K \varepsilon
$$

where $\sigma_{0}$ is constant for all grain orientations, as shown in Fig. 5(a).

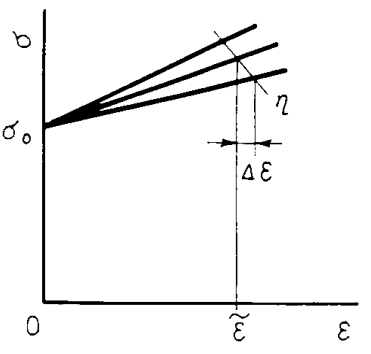

(a)

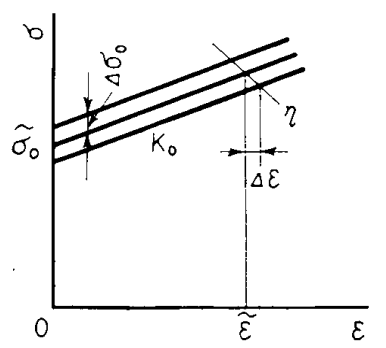

(d)

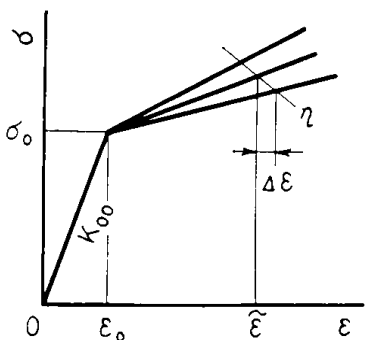

(b)

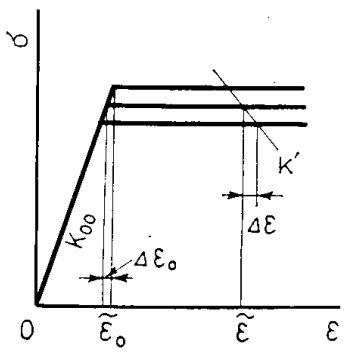

(e)

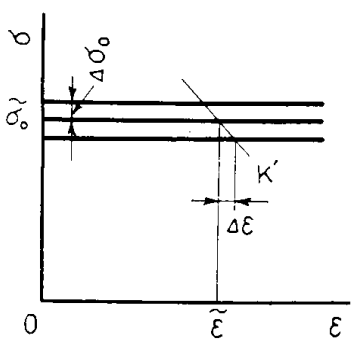

(c)

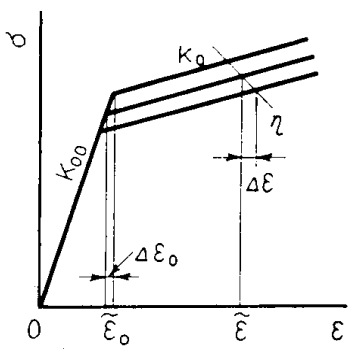

(f)

Fig. 5 Stress-strain diagram composed of combination of straight lines 
The deformation energy $W^{\prime}$ for the system of two grains without restriction between them is given from Eq. (9), similarly, as follows:

$$
\begin{aligned}
W^{\prime} & =\frac{1}{2}\left(W^{+}+W^{-}\right) \\
& =\sigma_{0} \tilde{\varepsilon}+\frac{1}{2}\left\{\widetilde{\left(\sigma-\sigma_{0}\right)} \tilde{\varepsilon}-|\Delta \sigma||\Delta \varepsilon|\right\} \\
& =\frac{1}{2}\left\{\sigma_{0} \tilde{\varepsilon}+\widetilde{K}_{\sigma} \tilde{\varepsilon}^{2}+(1-\eta)^{2} \frac{\left(\Delta K_{\varepsilon}\right)^{2}}{\widetilde{K}_{\varepsilon}} \tilde{\varepsilon}^{2}\right\}
\end{aligned}
$$

For polycrystals, from Eq. (10), we have

$$
W=\frac{1}{2}\left\{\sigma_{0} \tilde{\varepsilon}+\tilde{K}_{o} \tilde{\varepsilon}^{2}+(1-\eta)^{2} \frac{\widehat{\Delta \bar{K}_{\varepsilon}} \widetilde{K}_{\varepsilon}^{2}}{\widetilde{K}_{\varepsilon}^{2}}\right\}
$$

Next, the increase of energy at the boundary region is given from Eq. (12) by ${ }^{(1)}$,

$$
W_{b}=\widetilde{P}_{1} \eta^{2}\left(1-\frac{2}{3} \eta\right) \frac{\widetilde{\Delta K_{i j}^{2}}}{\widetilde{K}_{\varepsilon}} \tilde{\varepsilon}^{2}
$$

where $\widetilde{\Delta K_{i j}{ }^{2}}$ is the mean value of $\Delta K_{i j}{ }^{2}$ for all grains.

The total deformation energy $W_{t}$ is given from Eqs. (17), (20) and (21), and the value $\eta$ which minimizes $W_{t}$ is given from $\partial W_{t} / \partial \eta=0$.

$$
\eta=\min \left(1, \frac{\widetilde{\Delta K_{\varepsilon}^{2}}}{2 \widetilde{P_{1} \widetilde{\Delta K_{i j}{ }^{2}}}}\right)
$$

The result is in agreement with the previous case that the stress-strain relation is expressed with straight line starting from the origin (1). Consequently the value of $\sigma_{0}$ does not affect the distribution of $\eta$ in the polycrystals.

3.2 The case that the stress-strain relation is expressed by

$$
\left.\begin{array}{ll}
\sigma=K_{00} \varepsilon & \left(\varepsilon \leqq \varepsilon_{0}\right) \\
\sigma=\sigma_{0}+K\left(\varepsilon-\varepsilon_{0}\right) & \left(\varepsilon \geqq \varepsilon_{0}\right)
\end{array}\right\}
$$

where $K_{00}$ is a constant, as shown in Fig. 5(b).

The deformation energy $W$ is obtained from Eq. ( 7 ) as follows.

$$
\begin{aligned}
W & =\frac{1}{2}\left\{\sigma_{0} \tilde{\varepsilon}+\widetilde{K}_{\sigma}\left(\tilde{\varepsilon}-\varepsilon_{0}\right)^{2}\right. \\
& \left.+(1-\eta)^{2} \frac{\left(\widetilde{\Delta K_{\varepsilon}}\right)^{2}}{\widetilde{K}_{\varepsilon}}\left(\widetilde{\varepsilon}-\varepsilon_{0}\right)^{2}\right\}
\end{aligned}
$$

It will be shown easily that the same expression for $\eta$ in Eq. (22) is obtainable from Eq. (24).

3.3 The case that the stress-strain relation is expressed by

$$
\sigma=\tilde{\sigma}_{0}+\Delta \sigma_{0}
$$

irrespective of the strain as shown in Fig. 5(c).

It is inadequate to adopt the constraint ratio $\eta$ in this case, for $\eta$ is defined with the difference of the strains [Eq. (4)]. So, the inclination $K^{\prime}$ in Fig. 2 is used as the substitute for $\eta$. The mean value $\tilde{\sigma}_{0}$ of stress is independent of $K^{\prime}$ as shown in Fig. 5(c).

The deformation energy $W^{\prime}$ of the two grains is given from Eq. (9) by

$$
W^{\prime}=\widetilde{\sigma}_{0} \tilde{\varepsilon}-\left|\Delta \sigma_{0}\right||\Delta \varepsilon|
$$

It is evident from Fig. 5 (c) that $\left|\Delta \sigma_{0}\right|=-K^{\prime}|\Delta \varepsilon|$. Then, the deformation energy $W$ of polycrystals is given from Eq. (10) by,

$$
W=\widetilde{\sigma}_{0} \tilde{\varepsilon}+\frac{1}{K^{\prime}} \widetilde{\left(\Delta \sigma_{0}\right)^{2}}
$$

where $K^{\prime}$ is negative. Consequently, if the restriction between grains is absent, the energy $W$ becomes small as the inclination $\left|K^{\prime}\right|$ decreases to zero which corresponds to the condition of constant stress (Considering from the physical meaning of $\left.K^{\prime}, \quad\left|K^{\prime}\right| \geqq\left|\Delta \sigma_{0}\right| / \widetilde{\varepsilon}\right)$. The energy increase $\Delta W$ owing to the restriction at the boundary is given corresponding to the change in the inclination from $K^{\prime}$ to $K_{1}{ }^{\prime}$ as follows.

$$
\Delta W=\bar{W}\left(K_{1}^{\prime}\right)-\bar{W}\left(K^{\prime}\right)=\left(\frac{1}{K_{1}{ }^{\prime}}-\frac{1}{K^{\prime}}\right) \Delta \sigma_{0 i j^{2}}
$$

where $\Delta \sigma_{0 i j}$ is the difference of $\Delta \sigma_{0}$ between the two grains. If the distribution of strain is assumed to be linear in the boundary region, the change in $1 / K^{\prime}$ becomes also linear. For the simplicity of the calculation, let us define $\kappa$ by $\kappa=1 / K^{\prime}(\kappa<0)$. Then, similar analysis is available for $\kappa$ as well as the above $\eta$. The energy increase $W_{b}^{\prime}$ in the boundary region is given by

$$
W_{b}^{\prime}=2 P_{1} \int_{\kappa}^{0}\left(\kappa_{1}-\kappa\right) \Delta \sigma_{0 i j^{2}} d \kappa_{1}=P_{1} \kappa^{2} \Delta \sigma_{0 i j^{2}}
$$

Substituting $W_{b}^{\prime}$ into Eq. (16), the energy increase $W_{b}$ for the whole polycrystal is given by

$$
W_{b}=\widetilde{P}_{1} \kappa^{2} \widetilde{\Delta \sigma_{0 i j^{2}}}=\tilde{P}_{1} \frac{1}{K^{\prime 2}} \widetilde{\Delta \sigma_{0 i j^{2}}}
$$

Substituting Eqs. (26) and (27) into Eq. (17), the total deformation energy $W_{t}$ is obtained. The value $K^{\prime}$ which minimizes $W_{t}$ is given from $\partial W_{t} / \partial \kappa=0$, as follows:

$$
K^{\prime}=-\underbrace{2 \widetilde{P}_{1} \sigma_{0 i j^{2}}}_{\widetilde{\Delta} \sigma_{0}^{2}}
$$

3.4 The case that the stress-strain relation is expressed by

$\sigma=\sigma_{0}+K_{0} \varepsilon \cdots$

where only $\sigma_{0}$ is dependent on the grain orientation, as shown in Fig. 5(d).

In this case, the mean value $\widetilde{K}$ is independent of $\eta$, i.e. $\widetilde{K}=\widetilde{K}_{\sigma}=\widetilde{K}_{\varepsilon}=K_{0}$. The deformation energy $W^{\prime}$ is given from Eq. (9).

$$
\begin{aligned}
W^{\prime} & =\frac{1}{2}\left[\left(\tilde{\sigma}_{0}-\Delta \sigma_{0}\right)(\tilde{\varepsilon}+\Delta \varepsilon)+\frac{1}{2} K_{0}(\tilde{\varepsilon}+\Delta \varepsilon)^{2}\right. \\
& \left.+\left(\tilde{\sigma}_{0}+\Delta \sigma_{0}\right)(\tilde{\varepsilon}-\Delta \varepsilon)+\frac{1}{2} K_{0}(\tilde{\varepsilon}-\Delta \varepsilon)^{2}\right]
\end{aligned}
$$

Substituting $\Delta \varepsilon=\eta \Delta \varepsilon_{\max }=\eta \Delta \sigma_{0} / K_{0}$ and the above equation into Eq. (10), the deformation energy $W$ for the whole polycrystal without restriction at the boundary is expressed as 


$$
W=\tilde{\sigma}_{0} \tilde{\varepsilon}+\frac{1}{2} K_{0} \tilde{\varepsilon}^{2}+\eta\left(\frac{\eta}{2}-1\right) \frac{\widetilde{\Delta \sigma_{0}^{2}}}{K_{0}}
$$

It follows from Eq. (30) that $W$ takes its minimum value at $\eta=1$.

The energy increase owing to restriction at the boundary is given from Eqs. (30) and (12), as follows:

$$
\Delta W=\left(\frac{1}{2} \eta^{\prime 2}-\eta^{\prime}-\frac{1}{2} \eta^{2}+\eta\right) \frac{\left(\Delta \sigma_{0 i j}\right)^{2}}{K_{0}}
$$

The energy increase $W_{b}$ in the grain boundary region for the whole polycrystal is given by substituting the above equation and Eq. (15) into Eq. (16).

$$
W_{b}=\widetilde{P}_{1} \eta^{2}\left(1-\frac{2}{3} \eta\right) \frac{\widetilde{\left(\Delta \sigma_{0 i j}\right)^{2}}}{K_{0}}
$$

Finally, substituting Eqs. (30) and (31) into Eqs. (17) and (18), the value of $\eta$ which minimizes the deformation energy $W_{t}$ of polycrystals is obtained:

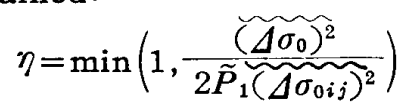

3.5 The case that stress-strain relation is expressed by

$$
\sigma=K_{00}\left(\tilde{\varepsilon}_{0}+\Delta \varepsilon_{0}\right)
$$

where only $\Delta \varepsilon_{0}$ is dependent on the grain orientation as shown in Fig. 5(e). (It is assumed that $\left.\varepsilon>\widetilde{\varepsilon}_{0}+\Delta \varepsilon_{0}\right)$.

The deformation energy $W^{\prime}$ for the two grains is written from Eq. (9) as follows.

$$
\begin{aligned}
& W^{\prime}=\frac{1}{2}\left[\frac{1}{2} K_{00}\left(\tilde{\varepsilon}_{0}+\Delta \varepsilon_{0}\right)^{2}+K_{00}\left(\widetilde{\varepsilon}_{0}+\Delta \varepsilon_{0}\right)(\tilde{\varepsilon}-\Delta \varepsilon\right. \\
& \left.\quad-\widetilde{\varepsilon}_{0}-\Delta \varepsilon_{0}\right)+\frac{1}{2} K_{00}\left(\tilde{\varepsilon}_{0}-\varepsilon_{0}\right)^{2}+K_{00}\left(\widetilde{\varepsilon}_{0}-\Delta \varepsilon_{0}\right) \\
& \left.\quad \times\left(\tilde{\varepsilon}+\Delta \varepsilon-\widetilde{\varepsilon}_{0}+\Delta \varepsilon_{0}\right)\right]
\end{aligned}
$$

Substituting $\Delta \varepsilon=-K_{00} \Delta \varepsilon_{0} / K^{\prime}$ and the above equation into Eq. (10), the deformation energy $W$ is obtained.

$$
W=\frac{1}{2} K_{00}\left[2 \widetilde{\varepsilon}_{0} \tilde{\varepsilon}-\widetilde{\varepsilon}_{0}^{2}+\frac{2 K_{00}}{K^{\prime}} \Delta \varepsilon_{0}^{2}-\Delta \varepsilon_{0}^{2}\right]
$$

The value of $W$ decreases with the decrease of $K^{\prime}$ as $K^{\prime}$ is negative. \{Considering from the physical meaning of $\left.K^{\prime},\left|K^{\prime}\right| \geqq\left|\Delta \sigma_{0}\right| /\left(\bar{\varepsilon}-\bar{\varepsilon}_{0}-\Delta \varepsilon_{0}\right)\right\}$. The energy increase $\Delta W$ in the boundary region is given from Eq. (12) by

$$
\Delta W=\bar{W}\left(K_{1}{ }^{\prime}\right)-\bar{W}\left(K^{\prime}\right)=K_{00}{ }^{2} \Delta \varepsilon_{0 i}{ }^{2}\left(\frac{1}{K_{1}{ }^{\prime}}-\frac{1}{K^{\prime}}\right)
$$

Putting $\kappa=1 / K^{\prime}$ (c.f. section $3 \cdot 3$ ), the energy $W_{b}{ }^{\prime}$ in the boundary region is given by

$$
W_{b}^{\prime}=2 P_{1} \int_{x}^{0}\left(\kappa_{1}-\kappa\right) K_{00}{ }^{2} \Delta \varepsilon_{0 i}{ }^{2} d \kappa_{1}
$$

Substituting $W_{b}^{\prime}$ into Eq. (16), we have

$$
W_{b}=\tilde{P}_{1} \kappa^{2} K_{00}{ }^{2} \widetilde{\Delta \varepsilon_{0 i j^{2}}}=\widetilde{P}_{1} \frac{K_{00}^{2}}{K^{\prime 2}} \varepsilon_{0 i j^{2}}
$$

Finally, substituting Eqs. (34) and (35) into Eqs. (17) and (18), the value $K^{\prime}$ which minimizes the total deformation energy $W_{t}$ is obtained.

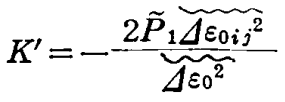

3.6 The case that the stress-strain relation is expressed by

$$
\sigma=K_{00}\left(\tilde{\varepsilon}_{0}+\Delta \varepsilon_{0}\right)+K_{0}\left(\varepsilon-\tilde{\varepsilon}_{0}-\Delta \varepsilon_{0}\right)
$$

where only $\Delta \varepsilon_{0}$ is dependent on the grain orientation as shown in Fig. 5(f) (It is assumed that $\left.\varepsilon>\widetilde{\varepsilon}_{0}+\Delta \varepsilon_{0}\right)$.

The energy $W^{\prime}$ is obtained from Eq. (9);

$$
\begin{aligned}
& W^{\prime}=\frac{1}{2}\left[K_{0}\left(\widetilde{\varepsilon}^{2}+\tilde{\varepsilon}_{0}^{2}-2 \widetilde{\varepsilon} \tilde{\varepsilon}_{0}+\Delta \varepsilon^{2}-\Delta \varepsilon_{0}^{2}\right)\right. \\
& \left.+K_{00}\left(2 \widetilde{\varepsilon}_{0} \tilde{\varepsilon}-\tilde{\varepsilon}_{0}^{2}-2 \Delta \varepsilon_{0} \Delta \varepsilon-\Delta \varepsilon_{0}^{2}\right)\right]
\end{aligned}
$$

Substituting $\Delta \varepsilon=\eta K_{00} \Delta \varepsilon_{0} / K_{0}$ and the above equation into Eq. (10), the deformation energy $W$ is obtained.

$$
\begin{aligned}
W & =\frac{1}{2}\left[K_{0}\left\{\tilde{\varepsilon}^{2}+\widetilde{\varepsilon}_{0}^{2}-2 \tilde{\varepsilon} \widetilde{\varepsilon}_{0}+\left(\eta \frac{K_{00}{ }^{2}}{K_{0}{ }^{2}}-1\right) \widetilde{\Delta \varepsilon_{0}^{2}}\right\}\right. \\
& \left.+K_{00}\left\{2 \widetilde{\varepsilon}_{0} \tilde{\varepsilon}-\widetilde{\varepsilon}_{0}^{2}-\left(2 \eta \frac{K_{00}}{K_{0}}-1\right) \widetilde{\Delta \varepsilon_{0}^{2}}\right\}\right] \cdots \cdots
\end{aligned}
$$

The energy $W_{b}$ in the boundary region is derived from Eqs. (12), (15), (16) and (38);

$$
W_{b}=\widetilde{P}_{1} \eta^{2}\left(1-\frac{2}{3} \eta\right) \frac{K_{00}^{2}}{K_{0}} \widetilde{\Delta \varepsilon_{0 i} j^{2}}
$$

The value $\eta$ which minimizes the total deformation energy is given from Eqs. (17), (18), (38) and (39).

$$
\eta=\min \left(1, \frac{\widetilde{\Delta \varepsilon_{0}^{2}}}{2 \widetilde{P}_{1} \Delta \varepsilon_{0 i j^{2}}}\right)
$$

In those cases treated in the sections $3 \cdot 1$ to $3 \cdot 6$, it is assumed that the stress-strain relation is expressed with a combination of straight lines and only one parameter of them is dependent on the grain orientation. For more complicated cases of two parameters in stressstrain relation being dependent on the grain orientation, it may be difficult to express the result in a general analytical form, although the analysis may be possible in a similar way to the above.

\section{Deformation of polycrystals having exponential stress-strain relation}

\subsection{Basic equations of stress and strain}

It is assumed that the stress in an arbitrary grain is expressed with the following exponential function of the strain.

$$
\sigma=k \varepsilon^{n} \text {. }
$$

where $k$ and $n$ are the material constants. In the following analysis, it is assumed that $n$ is independent of the grain orientations, while $k$ is dependent on them.

Denoting the mean value of $k$ for polycrystals by $\widetilde{k}$, we have 


$$
k=\widetilde{k}+\Delta k=\widetilde{k}_{\varepsilon}+\Delta k_{\varepsilon}=\widetilde{k}_{\sigma}+\Delta k_{\sigma}
$$

The deviation $\Delta \sigma$ of stress from the mean value is given from Eqs. (2) and (41).

$$
\Delta \sigma=\sigma-\tilde{\sigma}=k(\tilde{\varepsilon}+\Delta \varepsilon)^{n}-\widetilde{k} \widetilde{\varepsilon}^{n}
$$

If the second order of $\Delta \varepsilon$, is neglected assuming that $\tilde{\varepsilon} \gg \Delta \varepsilon$, we have

$$
\Delta \sigma \cong \Delta k \widetilde{\varepsilon}^{n}+(\widetilde{k}+\Delta k) n \tilde{\varepsilon}^{n-1} \Delta \varepsilon
$$

Considering the case of $\eta=1$ and putting $\Delta \sigma=0$ in Eq. (43), the maximum deviation of strain is given by

$$
\left(\Delta \varepsilon_{\max }\right)_{\sigma} \cong \frac{-\Delta k_{\sigma}}{k n} \tilde{\varepsilon}
$$

where $\Delta k_{\sigma}$ and $\left(\Delta \varepsilon_{\max }\right)_{\sigma}$ represent respectively the values of $\Delta k$ and $\Delta \varepsilon_{\max }$ under the constant stress condition. (When $\eta$ changes, the inclination $\widetilde{k}$ in Fig. 6 changes. Accordingly, the position of the point $O^{\prime}$ and the value of $\Delta \varepsilon_{\max }$ change slightly.) For an arbitrary value of $\eta$, Eq. (44) is replaced by

$$
\Delta \varepsilon_{\max } \cong \frac{-\Delta k}{k n} \widetilde{\varepsilon}
$$

Substituting Eq. (45) into Eq. ( 4 ), we have

$$
\Delta \varepsilon \cong \frac{-\eta \Delta k}{k n} \widetilde{\varepsilon}
$$

Substituting Eq. (46) into Eq. (43), $\Delta \sigma$ is given by

$$
\Delta \sigma \cong(1-\eta) \Delta k \tilde{\varepsilon}^{n}=(1-\eta) \Delta \sigma_{\max }
$$

If the constraint ratio $\eta$ is constant irrespective of the value $\Delta k$, the trace of the position of deformed grains on the stress-strain diagram becomes a curve, as shown in Fig. 6. The equation of the curve is obtained from Eqs. (46) and (47).

$$
\Delta \sigma=-\frac{1-\eta}{\eta} n \widetilde{k}_{\varepsilon} \tilde{\varepsilon}^{n-1} \Delta \varepsilon
$$

Let us denote the point of intersection of the above curve and $\sigma=k_{\varepsilon} \varepsilon^{n}$ curve by $\mathrm{O}^{\prime}$, and the inclinations of the respective curves at the point $\mathrm{O}^{\prime}$ by $K^{\prime}$ and $\tilde{K}$.

$$
\begin{aligned}
K^{\prime} & =\left.\frac{\partial(\Delta \sigma)}{\partial(\Delta \varepsilon)}\right|_{\Delta \varepsilon=0}=-\frac{1-\eta}{\eta} n \widetilde{k}_{\varepsilon} \widetilde{\varepsilon}^{n-1} \\
& =-\frac{1-\eta}{\eta} \widetilde{K} \quad \ldots \ldots \ldots \ldots \ldots \ldots \ldots \ldots \ldots \ldots \ldots
\end{aligned}
$$

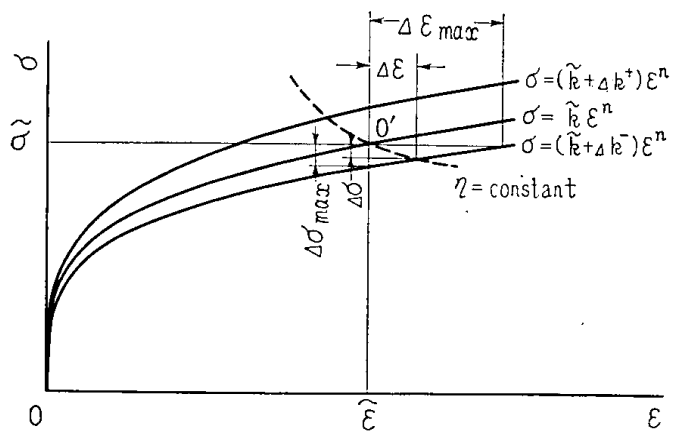

Fig. 6 Stress-strain relation of exponential type
It follows that Eq. (49) has the same form as Eq. ( 6 ).

\subsection{Mean value $\tilde{k}$ of $\boldsymbol{k}$}

The mean value $\widetilde{k}$ of $k$ for the whole polycrystals in the case of an arbitrary value of the constraint ratio $\eta$ is defined as follows [c.f. Eq. (3)].

$$
\widetilde{\sigma}=\widetilde{k} \widetilde{\varepsilon}^{n}
$$

From Eq. (41), we have

$$
\int\left(\sigma-k \varepsilon^{n}\right) d \Omega=0
$$

The mean values $\widetilde{k}_{\varepsilon}$ and $\widetilde{k}_{\sigma}$ of $k$ under the constant strain and the constant stress, respectively, are obtained from Eqs. ( 3 ) and (51).

$$
\begin{aligned}
& \widetilde{k}_{\varepsilon}=\int k d \Omega \ldots \ldots \ldots \ldots . . . \\
& \widetilde{k}_{\sigma}=1 /\left[\int \frac{1}{k^{1 / n}} d \Omega\right]^{n}
\end{aligned}
$$

Next, it is necessary to calculate the mean value $\widetilde{k}$ for an arbitrary value of the constraint ratio $\eta$. Substituting Eqs. (2.) and (3) into Eq. (51), we have

$$
\widetilde{\sigma}-\int k(\tilde{\varepsilon}+\Delta \varepsilon)^{n} d \Omega=0
$$

Neglecting the second and higher orders of $\Delta \varepsilon$, and substituting Eqs. (4) and (52) into the above equation, we have

$$
\widetilde{\sigma}-\tilde{k}_{\varepsilon} \tilde{\varepsilon}^{n}-n \eta \widetilde{\varepsilon}^{n-1} \int k \Delta \varepsilon_{\max } d \Omega \cong 0
$$

Namely,

$$
\widetilde{k} \cong \widetilde{k}_{\varepsilon}+n \eta \frac{1}{\tilde{\varepsilon}} \int k \Delta \varepsilon_{\max } d \Omega
$$

If we consider the condition of the constant stress, we have

$$
\widetilde{k}_{\sigma} \cong \widetilde{k}_{\varepsilon}+n \frac{1}{\tilde{\varepsilon}} \int k\left(\Delta \varepsilon_{\max }\right)_{\sigma} d \Omega
$$

Substituting Eq. (55) and approximating that $\left(\Delta \varepsilon_{\max }\right)_{\sigma} \cong \Delta \varepsilon_{\max }$, Eq. (54) reduces to

$$
\widetilde{k} \cong \widetilde{k}_{\sigma}+(1-\eta)\left(\widetilde{k}_{\varepsilon}-\widetilde{k}_{\sigma}\right)
$$

Substituting $k=\tilde{k}_{\varepsilon}+\Delta k_{\varepsilon}$ and $\int \Delta \varepsilon_{\max } d \Omega=0$ into Eq. (55), we have

$$
\widetilde{k}_{\varepsilon}-\widetilde{k}_{\sigma}=-n \frac{1}{\tilde{\varepsilon}} \int \Delta k_{\varepsilon}\left(\Delta \varepsilon_{\max }\right)_{\sigma} d \Omega
$$

On the other hand, from Eq. (44)

$$
\frac{\left(\Delta \varepsilon_{\max }\right)_{0}}{\tilde{\varepsilon}} \cong-\frac{\Delta k_{\sigma}}{n k} \cong-\frac{\Delta k_{\varepsilon}}{n \widetilde{k}_{\varepsilon}}
$$

(The right side of Eq. (58) is derived from Eqs. (62) and (63), which is to be mentioned afterwards). Substituting Eq. (58) into Eq. (57), we have

$$
\widetilde{k}_{\varepsilon}-\widetilde{k}_{\sigma} \cong \frac{1}{\widetilde{k}_{\varepsilon}} \widetilde{\left(\Delta k_{\varepsilon}\right)^{2}}
$$

Finally, substituting Eq. (59) into Eq. (56), $\widetilde{k}$ is represented as follows. 


$$
\widetilde{k}=\widetilde{k}_{\sigma}+(1-\eta) \frac{\left(\widetilde{\Delta k_{\varepsilon}}\right)^{2}}{\widetilde{k}_{\varepsilon}}
$$

4.3 Deformation of polycrystals without restriction at the grain boundary

At first, an imaginary case with no mutual restriction at the grain boundary is discussed. The deformation energy $W$ of the system composed of two grains having the coefficients $k^{+}$and $k^{-}$as shown in Fig. $7(\mathrm{a})$ is considered.

$$
\left.\begin{array}{l}
k^{+}=\bar{k}+\Delta k^{+}, \quad k^{-}=\bar{k}+\Delta k^{-} \\
k^{+}-k^{-}=\Delta k^{+}-\Delta k^{-}=2 \Delta k_{\varepsilon}
\end{array}\right\}
$$

where $\Delta k^{+}>0$ and $\Delta k^{-}<0$, and $\bar{k}$ is the mean value of $k$ for the two grains. Fig. $7(\mathrm{~b})$ is an enlarged view of the encircled region of Fig. $7(a)$. From Fig. 7(b), we have

$$
\left.\begin{array}{l}
\Delta k^{+}=\Delta k_{\varepsilon}+\left(\bar{k}_{\varepsilon}-\bar{k}\right) \\
\Delta k^{-}=-\Delta k_{\varepsilon}+\left(\bar{k}_{\varepsilon}-\bar{k}\right)
\end{array}\right\}
$$

From Eqs. (56), (59) and (62)

$$
\begin{aligned}
& \Delta k^{+}+\Delta k^{-}=2\left(\bar{k}_{\varepsilon}-\bar{k}\right) \\
& \cong 2 \eta\left(\bar{k}_{\varepsilon}-\bar{k}_{\sigma}\right)=2 \eta \frac{\left(\Delta k_{\varepsilon}\right)^{2}}{\bar{k}_{\varepsilon}}
\end{aligned}
$$

Consequently, the deformation energy $W^{\prime}$ of the system of the two grains is given by

$$
\begin{aligned}
W^{\prime} & =\frac{1}{2}\left[W^{+}+W^{-}\right] \\
& =\frac{1}{2}\left[\int_{0}^{\bar{\varepsilon}-|\Delta \varepsilon|}\left(\bar{k}+\Delta k^{+}\right) \varepsilon^{n} d \varepsilon\right. \\
& \left.+\int_{0}^{\bar{\varepsilon}+|\Delta \varepsilon|}\left(\bar{k}+\Delta k^{-}\right) \varepsilon^{n} d \varepsilon\right] \\
& =\frac{1}{2(n+1)}\left[\left(\bar{k}+\Delta k^{+}\right)(\bar{\varepsilon}-|\Delta \varepsilon|)^{n+1}\right. \\
& \left.+\left(\bar{k}+\Delta k^{-}\right)(\bar{\varepsilon}+|\Delta \varepsilon|)^{n+1}\right]
\end{aligned}
$$

Assuming $\bar{\varepsilon} \gg \Delta \varepsilon$ and substituting Eqs. (4), (58), (60), (61) and (62) into the above equation, we have

$$
\begin{aligned}
W^{\prime} & \cong \frac{1}{2}\left[\frac{2}{n+1} \bar{k} \bar{\varepsilon}^{n+1}-\left(\Delta k^{+}-\Delta k^{-}\right) \bar{\varepsilon}^{n}|\Delta \varepsilon|\right. \\
& \left.+n \bar{k} \bar{\varepsilon}^{n-1}|\Delta \varepsilon|^{2}+\frac{1}{n+1}\left(\Delta k^{+}+\Delta k^{-}\right) \bar{\varepsilon}^{n+1}\right] \\
& \cong \frac{1}{n+1}\left[\bar{k}_{\sigma}+(1-\eta) \frac{\left(\Delta k_{\varepsilon}\right)^{2}}{\bar{k}_{\varepsilon}}\right] \bar{\varepsilon}^{n+1} \\
& -\Delta k_{\varepsilon} \bar{\varepsilon}^{n}\left|\frac{\eta \Delta k_{\varepsilon}}{n \bar{k}_{\varepsilon}} \bar{\varepsilon}\right|+\frac{1}{2} n \bar{k} \bar{\varepsilon}^{n-1}\left[\frac{\eta \Delta k_{\varepsilon}}{n \bar{k}_{\varepsilon}} \bar{\varepsilon}^{2}\right. \\
& +\frac{1}{n+1} \eta \frac{\left(\Delta k_{\varepsilon}\right)^{2}}{k_{\varepsilon}} \bar{\varepsilon}^{n+1}=\frac{1}{n+1} \bar{k}_{\sigma} \bar{\varepsilon}^{n+1} \\
& +\frac{(n+1) \eta^{2}-2(n+1) \eta+2 n}{2 n(n+1)} \frac{\left(\Delta k_{\varepsilon}\right)^{2}}{\bar{k}_{\varepsilon}} \bar{\varepsilon}^{n+1}
\end{aligned}
$$

(In the above calculation, $\Delta k$ and $\tilde{k}$ are replaced approximately with $\Delta k_{\varepsilon}$ and $\tilde{k}_{\varepsilon}$, respectively, neglecting the terms of the second and higher orders of them). The deformation energy $W$ for the whole polycrystal is obtained by substituting Eq. (64) into Eq. (10) as follows.

$$
\begin{aligned}
& W \cong \frac{1}{n+1} \widetilde{k}_{\sigma} \widetilde{\varepsilon}^{n+1}+\frac{(n+1) \eta^{2}-2(n+1) \eta+2 n}{2 n(n+1)} \\
& \times \frac{\widetilde{\left(\Delta k_{\varepsilon}\right)^{2}}}{\widetilde{k}_{\varepsilon}}-\widetilde{\varepsilon}^{n+1}
\end{aligned}
$$

As the values of $\widetilde{k}_{\sigma}, \widetilde{k}_{\varepsilon}, \widetilde{\left(\Delta k_{\varepsilon}\right)^{2}}, \tilde{\varepsilon}^{n+1}$ and $n$ in Eq. (65) are positive, the value of $\eta$ which minimizes $W$ is obtained from $\partial W / \partial \eta=0$, and

$$
\eta=1
$$

It follows from Eq. (66) that the condition of stress constant is energetically stable if the mutual restriction between the grains is absent, which is the same conclusion as that of the polycrystals having a linear stress-strain relation, reported previously ${ }^{(1)}$.

4.4 Deformation of polycrystals with restriction at the grain boundary

Let us consider two neighbouring grains $I$ and $\mathrm{J}$ having coefficients $k_{i}$ and $k_{j}$ and denote their difference by $2 \Delta k_{i j}$.

$$
2 \Delta k_{i j}=k_{i}-k_{j}
$$

The difference $\Delta \varepsilon_{i j}$ of the strain between the two grains, is given from Eqs. (4), (58) and (67) by

$$
\begin{array}{r}
2 \Delta \varepsilon_{i j}=\Delta \varepsilon_{i}-\Delta \varepsilon_{j} \cong-\eta\left[\frac{\Delta k_{i}}{n \widetilde{k}_{\varepsilon}}-\frac{\Delta k_{j}}{n \widetilde{k}_{\varepsilon}}\right] \tilde{\varepsilon} \\
=-2 \eta \frac{\Delta k_{i j}}{n \widetilde{k}_{\varepsilon}} \tilde{\varepsilon} \ldots \ldots \ldots \ldots \ldots \ldots \ldots \ldots \ldots \ldots \ldots \ldots \ldots \ldots \ldots \ldots \ldots \ldots \ldots
\end{array}
$$

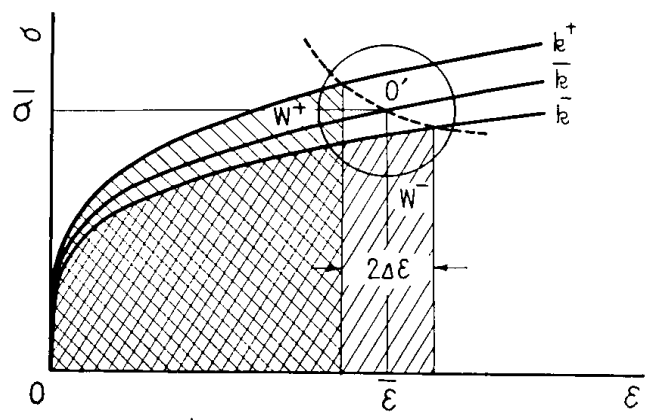

(a)

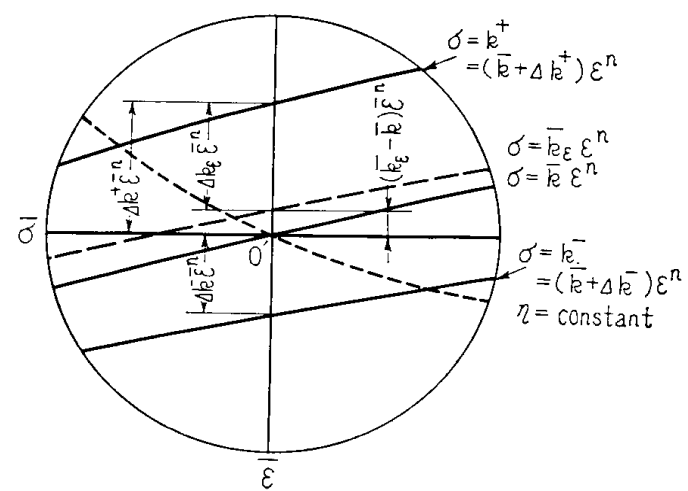

(b)

Fig. 7 Deformation energy and details of stressstrain curves 
Next, the energy increase owing to the mutual restriction at the grain boundary is taken into account. The energy increase $\Delta W$ corresponding to the decrease of the constraint ratio from $\eta$ to $\eta^{\prime}$ is given from Eq. (12):

$$
\Delta W \cong \frac{1}{2}\left[\left(1-\eta^{\prime}\right)^{2}-(1-\eta)^{2}\right] \frac{\left(k_{i j}\right)^{2}}{n \bar{k}_{\varepsilon}} \bar{\varepsilon}^{n+1} \ldots . . .(69)
$$

It may be natural to assume that the width $\Delta l$ of the grain boundary region is proportional to the difference of strain $2 \Delta \varepsilon_{i j}$ (Fig. 3), i.e. $\Delta l=$ $2 P^{\prime} \Delta \varepsilon_{i j}$, where $P^{\prime}$ is a coefficient. From Eqs. (13) and (68), we have

$$
P=P^{\prime} \frac{\eta\left|\Delta k_{i j}\right| \tilde{\varepsilon}}{n \widetilde{k}_{\varepsilon} l}
$$

Eq. (70) describes the deformation behavior in the boundary region more definitely than Eq. (14).

The energy $W_{b}$ in the grain boundary region for the whole polycrystal is obtained from Eqs. (14), (15), (16), (69) and (70) as follows.

$$
W_{b} \cong \widetilde{P}^{\prime} \eta^{2}\left(1-\frac{2}{3} \eta\right) \frac{\mid \widetilde{\left.\Delta k_{i j}\right|^{3}} \widetilde{\varepsilon}^{n+2}}{n^{2} \widetilde{k}_{\varepsilon}^{2} l}
$$

Finally, the total deformation energy $W_{t}$ for polycrystals is given by substituting Eqs. (65) and (71) into Eq. (17).

$$
\begin{aligned}
W_{t} & =\frac{1}{n+1} \widetilde{k}_{\sigma} \tilde{\varepsilon}^{n+1}+\frac{(n+1)}{\eta^{2}-2(n+1) \eta+2 n} \\
& \times \frac{\left(\widetilde{\left(\Delta k_{\varepsilon}\right)^{2}}\right.}{\widetilde{k}_{\varepsilon}} \widetilde{\varepsilon}^{n+1}+\widetilde{P}^{\prime} \eta^{2}\left(1-\frac{2}{3} \eta\right) \frac{\mid \widetilde{\left.\Delta k_{i j}\right|^{3} \widetilde{\varepsilon}^{n+2}}}{n^{2} \widetilde{k}_{\varepsilon}{ }^{2} l}
\end{aligned}
$$

Eq. (72) contains a third order term of $\eta$, which is schematically shown in Fig. 8. Considering that $0 \leqq \eta \leqq 1$, the value of $\eta$ which minimizes $W_{t}$ is given from $\partial W_{t} / \partial \eta=0$.

$$
\eta=\min \left(1, \frac{\left(\widetilde{\left.\Delta k_{\varepsilon}\right)^{2} n \widetilde{k}_{\varepsilon} l}\right.}{2 \widetilde{P^{\prime}\left|\widetilde{\Delta k}_{\imath j}\right|^{3} \bar{\varepsilon}}}\right)
$$

Eq. (73) expresses the constraint ratio of polycrystals having exponential stress-strain curves. The influence of the shape of stress-strain curve on $\eta$ is represented by $n$ and $\tilde{k}_{\varepsilon}$ in Eq. (73). When the second term in the parenthesis of Eq. (73) is smaller than $1, \eta$ increases proportionally with $n$ and $\tilde{k}_{\varepsilon}$. It is also said from Eq. (73)

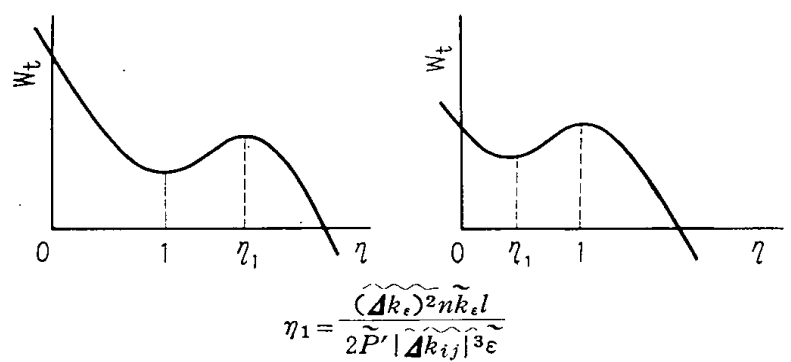

Fig. 8 Relation between deformation energy $W_{t}$ and restriction ratio $\eta$ of polycrystals (Schematic) that $\eta$ decreases with the decrease in the grain size $l$ and with the increase in the mean strain $\widetilde{\varepsilon}$. Thus, the effect of grain size on the deformation of polycrystals is expressed with $l$ in Eq. (73). (It is said ${ }^{(2)}$ that no theoretical study has been made on the effect of grain size on the deformation behavior of polycrystals.)

It is apparent that when the stress-Strain relation is expressed with

$$
\sigma=\sigma_{0}+K \varepsilon^{n}
$$

the expression of the constraint ratio $\eta$ has the same form as Eq. (73) \{cf. Eq. (19) in section $3 \cdot 1\}$.

From the above discussion, it follows that the portion in the polycrystals subjected to the highest deformation energy is the grain boundary region between the two adjacent grains which have the most widely different shapes of stressstrain curves each other, that is, which have the greatest value of $\Delta k_{i j}$. The observed crack initiation near the boundary ${ }^{(3)(4)}$ is supposed to be related to those much deformed region.

In the above discussion, the strain distribution in the boundary region is assumed to be linear. It is easily shown that, if the strain distributes in the form of a sine function in the boundary region, the coefficient of the second term in the parenthesis of Eq. (73) becomes 1.3 times larger than that in Eq. (73).

\section{Conclusions}

The deformation behavior of a polycrystalline metal composed of anisotropic crystals is analysed from energy aspect and particularly the effect of the shape of stress-strain curve is discussed. The results may be summarized as follows.

(1) The stress-strain relation is supposed to be (a) a combination of two straight lines and (b) an exponential curve, and for both cases the constraint ratio $\eta$ between grains is expressed in an analytical form.

(2) If there is no mutual restriction at the grain boundary, the deformation under the constant stress condition is energetically stable. On the other hand, the deformation behavior with mutual restriction between grains is dependent on the shape of stress-strain curves, as well as the anisotropy or the distribution of the grain orientations.

(3). Under the assumption that the width of the grain boundary region is proportional to the strain difference between the two grains, it is shown that the deformation of polycrystals changes its mode from constant stress to 
constant strain (i.e. $\eta$ decreases to zero), as the total strain becomes greater and as the grain size becomes smaller.

The author is indebted to Mr. T. Yamase for his kind help, to Prof. M. Oyane for his encouragement and to his wife for her help in translation.

\section{References}

(1) Abe, T., Bull. JSME. Vol. 12, No. $50(1969-4)$, p. 165.

(2) Hashin, Z., Appl. Mech. Rev., Vol. 17 (1964), p. 1.

(3) Clark, H. McI., Nature, Vol. 209 (1966), p. 193.

(4) Williams, H. D., et al, Phil. Mag., Vol. 13 (1966), p. 835 . 